\title{
Uniform Management of Data Acquisition Devices With XML
}

\author{
J. Gutleber, I. Magrans, M. Nafría, and L. Orsini
}

\begin{abstract}
A comprehensive model based on XML technologies to interface data acquisition hardware devices for configuration and control purposes is presented. The model builds upon the use of a unified syntax for describing hardware devices, configuration data, test results and control sequences. An implementation and an evaluation together with an application scenario are presented.
\end{abstract}

Index Terms-Configuration, data acquisition, extensible markup language (XML), fault diagnosis, front-end electronics, monitoring, testing.

\section{INTRODUCTION}

$\mathbf{T}$ HE preparation and operation of high-energy physics (HEP) experiments typically spans over a period of many years. During this time the hardware and software environments evolve toward the operational phase. Throughout all phases, integrators have to deal with system modifications [1].

Configuration and control systems, and their interfaces evolve through various design and evaluation cycles. In this context, several different hardware devices need to be configured, controlled, and tested.

Sequences containing read, write, test and exception handling operations for initialization of parameterization purposes must be defined. These sequences, for instance, are responsible for downloading firmware code and for setting tunable parameters like threshold values or parameters to compensate the accrued radiation damage. Many hardware modules are replicated and common procedures for configuration are required. Configuration is only one part of the problem: a mechanism to execute tests on hardware devices and for detecting and diagnosing faults is needed.

In such a heterogeneous and evolving environment, a considerable development effort is required to design and implement new interfaces, synchronize and integrate them with all other subsystems, and support the configuration and control of all parts. We believe that a uniform approach to manage the diverse interfaces and operations of the data acquisition devices would simplify the development of a configuration and control system and should save development time.

Manuscript received January 23, 2004. The work described in this paper is part of the thesis of I. Magrans, to be submitted in partial fulfillment of the requirements for the Ph.D. degree at the Universidad Autónoma de Barcelona.

J. Gutleber and L. Orsini are with CERN, CH-1211 Geneva 23, Switzerland.

I. Magrans is with CERN, CH-1211 Geneva 23, Switzerland, and also with Electronic Engineering Department, Universidad Autónoma de Barcelona, Barcelona, Spain (e-mail: ildefons.Magrans@cern.ch; ildefons@ieee.org).

M. Nafría is with Electronic Engineering Department, Universidad Autónoma de Barcelona. Spain.

Digital Object Identifier 10.1109/TNS.2004.829494
A uniform scheme would be advantageous for large installations, like those found in HEP experiments [2]-[4] due to the diversity of front-end electronic modules, in terms of configuration, functionality and multiplicity [5, pp. 14, 173].

\section{KEY REQUIREMENTS}

We propose to work toward an environment to define hardware devices and their behavior at a logical level. The approach facilitates the integration of various different hardware subsystems. The design should at least fulfill the following key requirements.

- Standardization: The environment must be based on standard technologies. This ensures a higher degree of support and tools availability than an in-house solution.

- Extensibility: A mechanism to define new commands and data for a given interface must exist, without the need to change either control or controlled systems that are not concerned with the modification.

- Platform independence: The specification of commands and configuration parameters must not impose a specific format of a particular operating system or hardware platform.

- Communication technology independence: Hardware devices are hosted by different subsystems that expose different capabilities and types of communication abilities. Choosing the technology that is most suitable for a certain platform must not require an overall system modification.

\section{A UNIFORM APPROACH FOR HARDWARE CONFIGURATION CONTROL AND TESTING}

Taking into account the above requirements, we present a model for the configuration, control and testing interface of data acquisition hardware devices. The model, shown in Fig. 1, builds upon two principles.

1) The use of the eXtensible Markup Language (XML [6]) as a uniform syntax for describing hardware devices, configuration data, test results and control sequences.

2) An interpreted, run-time extensible, high-level control language for these sequences that provides independence from specific hosts and interconnect systems to which devices are attached.

This model, as compared to other approaches [7], enforces the uniform use of XML syntax to describe configuration data, device specifications, and control sequences for configuration and control of hardware devices. This means that control sequences can be treated as data, making it easy to write scripts that manipulate other scripts and embed them into 


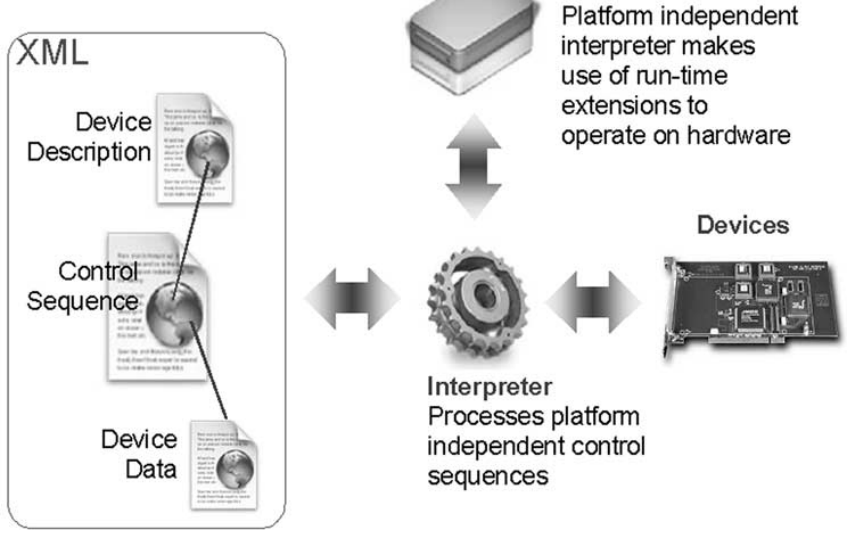

Fig. 1. Abstract description of the model.

other XML documents. In addition, the unified model makes it possible to use the same concepts, tools, and persistency mechanisms, which simplifies the configuration management of large projects [8].

\section{A. XML as a Uniform Syntax}

When designing systems composed of heterogeneous platforms and/or evolving systems, platform independence is provided by a uniform syntax, using a single data representation to describe hardware devices, configuration data, test results, and control sequences. A solution based on the XML syntax presents the following advantages.

- XML is a W3C (World Wide Web Consortium) nonproprietary, platform independent standard that plays an increasingly important role in the exchange of data. A large set of compliant technologies, like XML schema [9], DOM [10], XPath [11] are defined. In addition tools that support programming become available through projects like Apache [12].

- XML structures can be formally specified and extended, following a modularized approach, using an XML schema definition.

- XML documents can be directly transmitted using any kind of protocols including HTTP. In this case, SOAP [13], a XML based protocol, can be used.

- XML documents can be automatically converted into documentation artifacts by means of an XSLT transformation [14]. Therefore, system documentation can be automatically and consistently maintained.

- XML is widely used for nonevent information in HEP experiments: "XML is cropping up all over in online configuration and monitoring applications" [15].

On the other hand, XML has one big drawback: XML uses by default textual data representation, which causes much more network traffic to transfer data. Even BASE64 or UUencoded byte arrays are approximately 1.5 times larger than a binary format. Furthermore, additional processing time is required for translating between XML and native data representations. Therefore, the current approach is not well suited for devices generating abundant amount of real-time data, but is still valid for configuration, monitoring, and slow control purposes.

\section{B. XML Based Control Language}

A control language (Xseq: cross-platform sequencer) that processes XML documents to operate hardware devices has been syntactically and semantically specified. The language is XML based and has the following characteristics.

- Extensibility: The syntax has been formally specified using XML schema. A schema document contains the core syntax of the language, describing the basic structures and constraints on Xseq programs (e.g., variable declarations and control flow). The basic language can be extended in order to cope with user specific requirements. Those extensions are also XML schema documents, whose elements are instances of abstract elements of the core XML schema. This mechanism is one of the most important features of the language because it facilitates a modular integration of different user requirements and eases resource sharing (code and data). The usage and advantages of this feature will be discussed in Section VI.

- Imperative style: The language provides standard imperative constructs just like most other programming languages in order to carry out conditions, sequencing and iteration.

- Exception handling with error recovery mechanisms.

- Local execution of remote sequences with parameter passing by reference.

- Nontyped scoped variables.

Additional functionalities have been added to the core syntax in the form of modular XML schema extensions, in order to fit frequently encountered use cases in data acquisition environments:

- Transparent access to PCI and VME devices: This extension facilitates the configuration and control of hardware devices, following a common interface for both bus systems. This interface is designed to facilitate its extension in order to cope with future technologies.

- File system access.

- SOAP messaging: This allows inclusion of control sequences and configuration data into XML messages. The messages can be directly transported between remote hosts in a distributed programming environment.

- DOM and XPath interface to facilitate integration in an environment where software and hardware device configuration are fully XML driven.

- System command execution interface with redirected standard error and standard output to internal string objects.

In Fig. 2 an Xseq program is shown where basic features of the language are exemplified.

In Fig. 3 an example is given of how the hardware access is performed following the proposed model. Device specifications, configuration data and control sequences are XML documents. In this example, configuration data are retrieved through an XPath query from a configuration database.

\section{INTERPRETER DESIGN}

To enable code sharing among different platforms, we have chosen a pure interpreted approach that allows control 


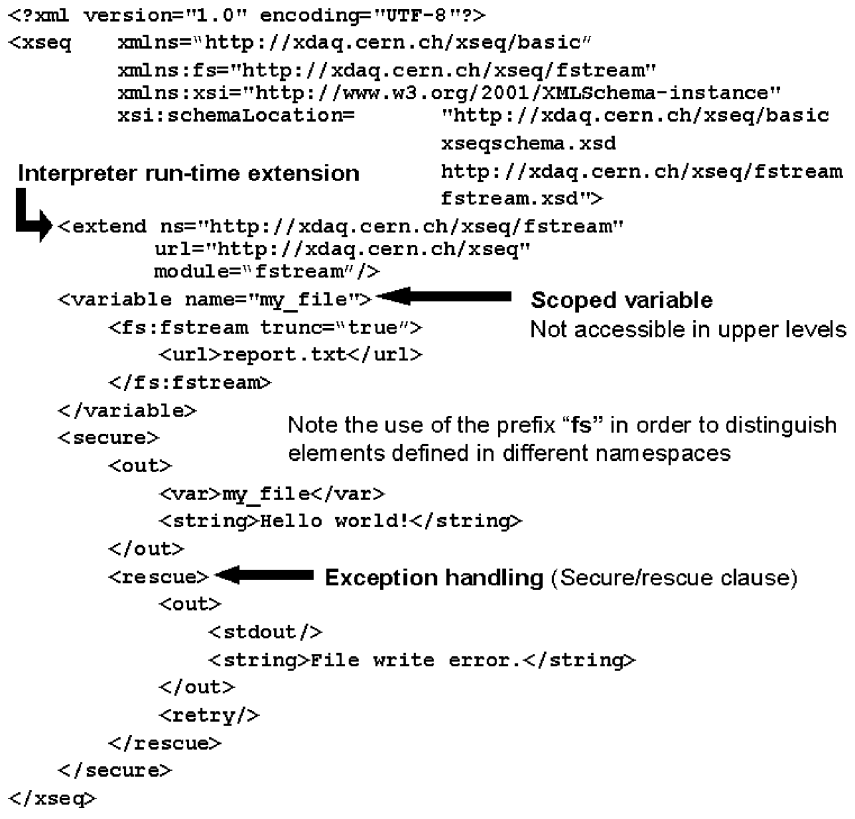

Fig. 2. Example program in Xseq exemplifying the basic features of the language.

sequences to run independently of the underlying platform in a single compile/execution cycle. In addition, the interpreted approach is characterized by small program sizes and an execution environment that provides controlled and predictable resource consumption, making it easily embeddable in other software systems.

An interpreter [16] for Xseq programs has been implemented in $\mathrm{C}++$ under Linux. The interpreter is available as a standalone tool and as a pluggable module for a programming framework for distributed data acquisition applications [17], [18]. The pattern of the interpreter is based on the following concepts.

- The source format is a DOM document already validated against the Xseq XML schema document and the required extensions. This simplifies interpreter implementation and separates the processing into two independent phases: 1) syntactic validation and 2) execution.

- Every XML command has a $\mathrm{C}++$ class representation that inherits from a single class named XseqObject.

- A global context accessible to all instruction objects. It contains: 1) the execution stack, which stores nonstatic variables; 2) the static stack, which stores static variables and is useful to retain information from previous executions; 3 ) the code cache, which maintains already validated DOM trees in order to accelerate the interpretation process; 4) the dynamic factory, which facilitates the interpreter run-time extension; and 5) debug information to properly trace the execution and to find errors.

\section{A. Polymorphic Structure}

Every class inherits from a single abstract class XseqObject, and it has information about how to perform its task. For example, the Xseq command $\langle i f\rangle$ is represented with the XseqIf class. This class inherits from the XseqObject class, and the execution algorithm is implemented in the overridden eval() method.
$\mathrm{C}++$ classes that implement the functionality of every language syntactic extension are compiled into shared libraries. They are dynamically linked to the running interpreter. Each run-time extension is associated with a syntactic language extension by means of the Xseq command $\langle$ extend $\rangle$. This facility makes it possible to separate syntactic language extensions, defined in XML schema modules, from the run-time interpreter extensions. Therefore, different implementations of a given extension can be mapped to one syntactic language extension.

\section{USE IN A DISTRIBUTED ENVIRONMENT}

The interpreter is also available as a pluggable module for a distributed data acquisition programming framework called XDAQ [19].

XDAQ includes an executive component that provides applications with the necessary functions for communication, configuration, control and monitoring. All configuration, control and monitoring commands can be performed through the SOAP/HTTP protocol.

In Fig. 4, the use of the interpreter in a XDAQ framework is shown. This is the basic building block that facilitates the deployment of the model in a distributed environment.

To operate this application, the user must provide configuration in XML format about the physical and logical properties of the system and its components. The configuration process defines the available web services as Xseq scripts.

Once the running application is properly configured, the client can send commands through SOAP messages. As a function of the received command, the corresponding Xseq script is executed. The SOAP message itself can be processed using the language extension to manipulate SOAP messages. Such functionality is useful when parameters must be remotely passed. Finally, every Xseq program ends by returning a SOAP message that will be forwarded by the executive to the client.

\section{HEP APPLICATION SCENARIO}

The architecture of a hypothetical hardware management system for a HEP experiment is shown in Fig. 5. Front-end electronic modules are quite heterogeneous in terms of configuration, functionality and multiplicity. In addition, the control software subsystem for every subdetector is independent from the others. Therefore, a diverse set of control software subsystems exists. This offers a heterogeneous set of interfaces that must be understood by a common configuration and control system.

The basic building block presented in Section $\mathrm{V}$ should be implemented for every different platform that plays the role of hardware module host or hardware management system supervisor. The configuration and control system is responsible for the hardware management system supervisor, which in turn manages the configuration of all hardware module hosts. An advantage of this approach is that both hardware and software configuration schemes are unified since the online software of the data acquisition system is fully XML driven.

Control sequences executed by the distributed control modules depend on a set of language extensions. The language can be augmented, following a modular approach, by means of 


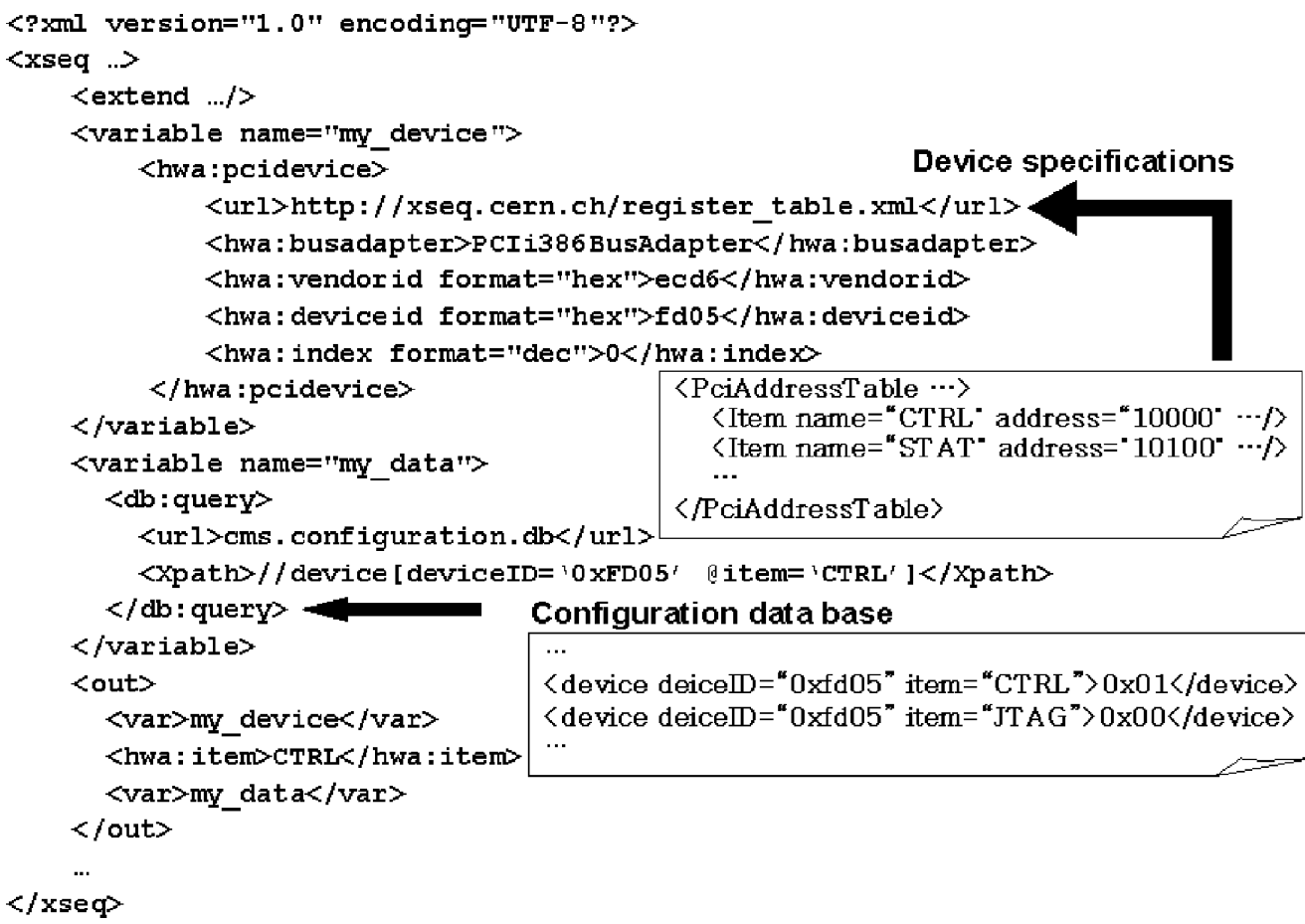

$\left\langle\right.$ device deice $\mathrm{D}=$ "0xfd05" item $={ }^{\text {"CTRL" }}$ " $>0 \times 01\langle/$ device $\rangle$ $\langle$ device deice $\mathrm{D}=$ "0xfd05" item $=$ "JT AG" $\rangle 0 \times 00\langle/$ device $\rangle$

Fig. 3. Example of a program in Xseq, which shows how the model is applied. Device specifications (register_table.xml), configuration data (retrieved from a configuration data base accessible through a XPath query) and control sequences are all of them based on uniform use of XML.

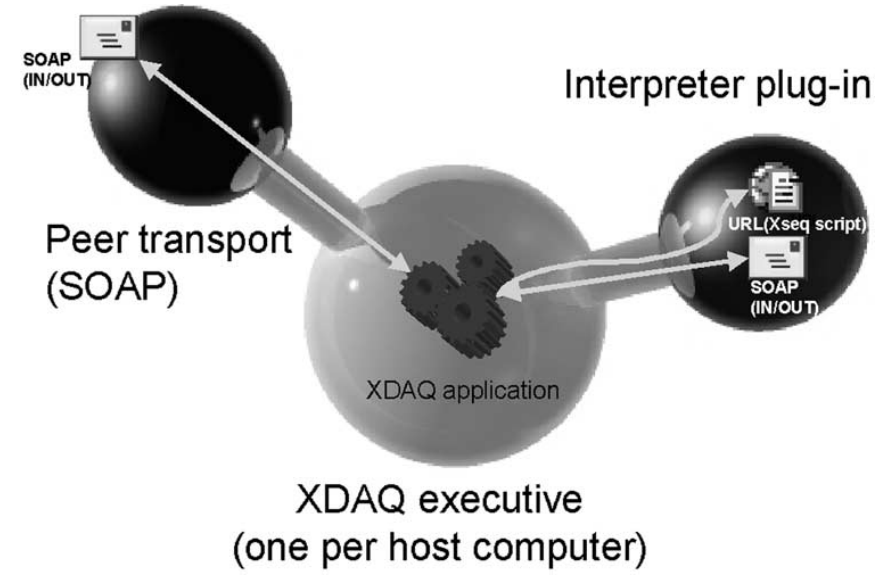

Fig. 4. Use of the interpreter in a XDAQ framework.

the XML schema technology. For a given language extension the interpreter associates a platform specific support. These extensions can be shared by several subsystems facilitating code sharing. Binding of the language extension to a specific implementation is performed at run-time. The above syntactic and semantic extensions are the mechanisms that allow the integration in the model of different control software APIs.

The homogeneous use of XML syntax to describe data, control sequences, and language extensions allows distributed storage of any of these documents that can be simply accessed through their URLs. Interpreter run-time extensions can be remotely linked and, therefore, a local binary copy is not necessary. This mechanism could be used in a final design in order to retrieve any of these documents from a trusted server.

A default SOAP extension of the control language makes it possible to manipulate, send, and receive SOAP messages.
Thus, code that accesses web services through a SOAP protocol can be written in Xseq.

\section{PeRformance COMPARISON}

Timing measurements have been performed on a desktop PC (Intel D845WN chipset) with a Pentium IV processor (1.8 GHz), $256 \mathrm{MB}$ SDRAM memory (133 MHz), running Linux Red Hat 7.2, with kernel version 2.4.9-31.1.

The main objective of this section is to present a comparison of the existing interpreter implementation with a Tcl interpreter [20], focusing on the overhead induced by the interpreter approach when accessing hardware devices. Tcl has been chosen as a reference because it is a well-established scripting language in the HEP community, and it shares many features with Xseq: it is simple, easily extensible and embeddable.

For both interpreters the same hardware access library (HAL [21]) has been used to implement the necessary extensions. This library has been also used to implement a $\mathrm{C}++$ binary version of the test program for reference purposes.

The test is a loop that reads $10^{6}$ consecutive memory positions of a memory module. In order to properly identify the interpreter overhead and to decouple it from the driver overhead, the real hardware access has been disabled and a dummy driver emulates all accesses. The results are shown in Table I.

The results indicate an overhead, which results from the interpreted approach that lies in the same order of magnitude as the $\mathrm{Tcl}$ interpreter. Execution times of Xseq can be further reduced with customized language extensions that encapsulate a specific macro behavior. For instance, a loop command with a fixed number of iterations has been implemented. This command reduces the timing of the test program to $5.3 \mu \mathrm{sec}$. 


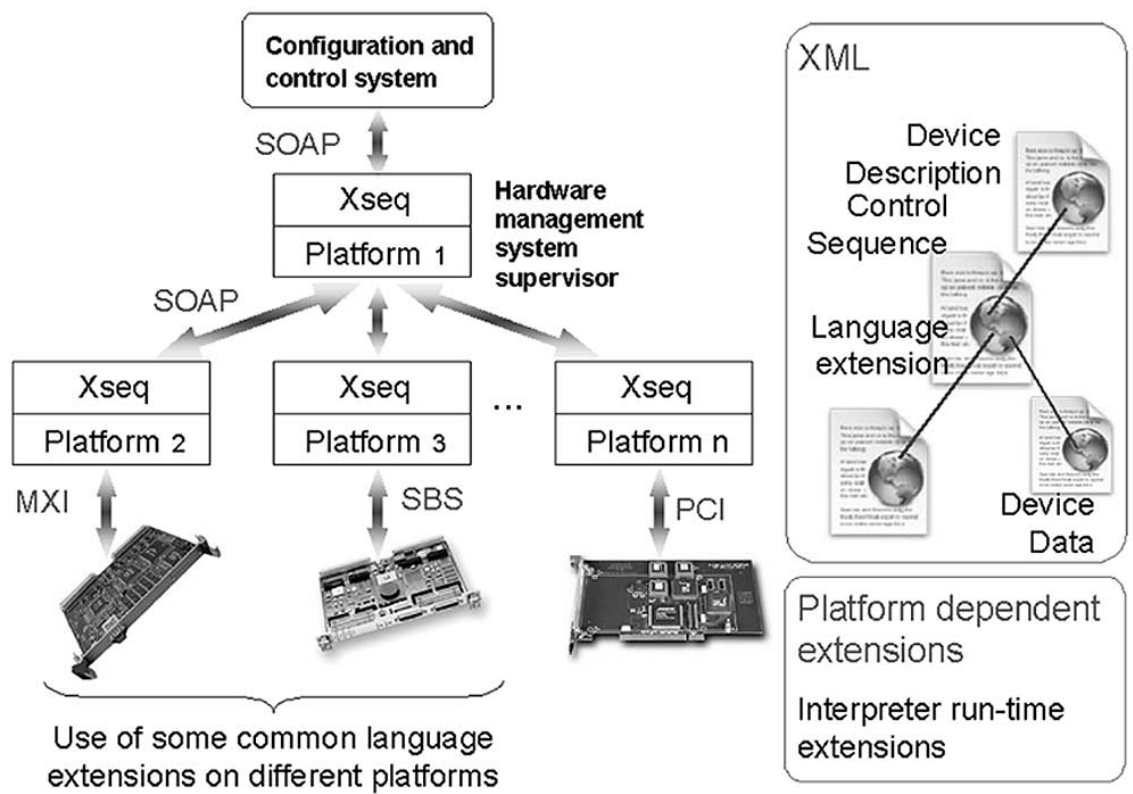

Fig. 5. Architecture of a hypothetical hardware management system.

TABLE I

COMPARISON OF AVERAGE EXECUTION TIMES ( $\mu \mathrm{sec} /$ Memory Read) FOR TCL XSEQ AND C++

\begin{tabular}{ccc}
\hline Tcl & Xseq & $\mathrm{C}++$ \\
\hline $16 \mu \mathrm{sec}$ & $16.9 \mu \mathrm{sec}$ & $2.63 \mu \mathrm{sec}$ \\
\hline
\end{tabular}

However, flexibility is reduced, because the macro command cannot be modified at run time.

\section{CONCLUSION}

This paper presents a uniform model based on XML technologies for the configuration, control and testing of data acquisition hardware. It matches well the extensibility and flexibility requirements of a long lifetime experiment that is characterized by an ever-changing environment. The model builds upon two points: 1) the use of XML for describing hardware devices, configuration data, test results, and control sequences and 2) an interpreted, run-time extensible, high-level control language for these sequences that provides independence from a specific host and from interconnect systems to which devices are attached.

An interpreter for such a language has been successfully implemented. This interpreter is run-time extensible and provides inspection commands similar to the GNU debugger. The current implementation of the interpreter is described along with the preliminary measurement in a Linux environment that shows an overhead, due to the interpreted approach, in the same order as the native $\mathrm{Tcl}$ interpreter.

The main goal of this work is to demonstrate that this model is a suitable approach for the reoccurring tasks of hardware device configuration, control and testing. An application scenario is presented and it is shown that integrating the presented model into a distributed programming framework, can form the architectural basis of a management system for DAQ hardware.

\section{ACKNOWLEDGMENT}

This study has been carried out in the context of the design project for the CMS experiment at CERN, Geneva, Switzerland. The authors gratefully acknowledge the possibility to benefit from the infrastructure provided within the Trigger and Data Acquisition Project as well as the subdetector application environments. We would like to thank all members of the collaboration for their continuous support and for the valuable exchange of ideas.

\section{REFERENCES}

[1] J. Gutleber, S. Murray, and L. Orsini, "Toward a homogeneous architecture for high-energy physics data acquisition systems," in Comp. Phy. Comm.: Elsevier cpc2578, to be published.

[2] "The Compact Muon Solenoid, CERN," CMS Coll., Tech. Proposal, 7, LHCC 94-38, 1995.

[3] "ATLAS Technical Proposal," ATLAS Collaboration, CERN/LHCC/94-43.

[4] “The CDF II Detector: Technical Design Report," CDF II Collaboration, FERMILAB-PUB-96/390-E, 1996.

[5] "The Trigger and Data Acquisition Project, Volume II, Data Acquisition \& High-Level Trigger, Technical Design Report," CMS Collaboration, CERN/LHCC 2002-26, CMS TDR 6.2, 2002.

[6] Extensible Markup Language (XML). W3C. [Online]. Available: http://www.w3.org/XML

[7] M. Elsing and T. Schorner-Sadenius, "Configuration of the ATLAS trigger system," in Proc. CHEP 2003, La Jolla, CA, 2003.

[8] Fletcher and J. Buckley, Implementing Configuration Management: Hardware, Software, and Firmware, 2nd ed. Los Alamitos, CA: IEEE Comp. Soc. Press, 1996.

[9] (2004) XML Schema. W3C. [Online]. Available: http://www.w3.org/ XML/Schema

[10] (2004) Document Object Model (DOM). W3C. [Online]. Available: http://www.w3.org/DOM/

[11] (2004) XML Path Language (XPath). W3C. [Online]. Available: http://www.w3.org/TR/xpath

[12] Apache Project (2004). [Online]. Available: http://xml.apache.org/

[13] (2004) Simple Object Access Protocol (SOAP). W3C. [Online]. Available: http://www.w3.org/TR/SOAP

[14] (2004) XSL Transformations (XSLT). W3C. [Online]. Available: http://www.w3.org/TR/xslt 
[15] G. Dubois-Felsman, Proc. Summary DAQ and Trigger at CHEP 2003, La Jolla, CA, 2003.

[16] S. N. Kamin, Programming Languages: An Interpreted-Based Approach. Reading, MA: Addison-Wesley, 1990.

[17] A. Luchetta and G. Manduchi, "General purpose architecture for real-time feedback control in nuclear fusion experiments," in Proc. 5th IEEE Real-Time Technology and Applications Symp. (RTAS99), Vancouver, BC, Canada, June 2-4, 1999.

[18] R. T. Hamilton, I. Scott, R. Claus, P. Grosso, M. E. Huffer, C. O'Grady, and J. J. Russel, "The BaBar data acquisition system," in Proc. 14th IEEE NPSS Real Time Conf., Santa Fe, NM, June 1999.
[19] J. Gutleber and L. Orsini, "Software architecture for processing clusters based on I2O," in Cluster Computing. New York: Kluwer, 2002, vol. 5, pp. 55-65.

[20] J. Ousterhout, Tcl and Tk Toolkit. Reading, MA: Addisson-Wesley, 1994.

[21] Documentation Links of the TriDAS Project [Online]. Available: http://cmsdoc.cern.ch/cms/TRIDAS/html/Documents.html 